\title{
Isolated superior mesenteric artery dissection: a rare cause of chronic abdominal pain
}

\begin{abstract}
Isolated superior mesenteric artery dissection (ISMAD) is unusual. We present a case of a 30-year-old man who had chronic abdominal pain for three months. Contrast-enhanced computed tomography (CECT) revealed type B superior mesenteric artery dissection (SMAD) without thrombus. The patient was managed conservatively.
\end{abstract}

Keywords: isolated superior mesenteric artery dissection, chronic abdominal pain, endovascular management
Volume 8 Issue I - 2017

\author{
Vipul D Yagnik \\ Consultant Endoscopic and laparoscopic surgeon, Ronak Endo- \\ laparoscopy and general surgical hospital, India
}

\begin{abstract}
Correspondence: Vipul Yagnik, Consultant Endoscopic and laparoscopic surgeon, Ronak Endo-laparoscopy and general surgical hospital, 77, Siddhraj Nagar, Rajmahal Road, Patan-384265, Gujarat, India, Email vipul.yagnik@gmail.com
\end{abstract}

\section{Introduction}

Isolated superior mesenteric artery dissection (ISMAD) is rare. In a series of 6,666 autopsies, the incidence of superior mesenteric artery dissection (SMAD) was $0.06 \%$. There have been 323 cases of ISMAD reported in the literature. ${ }^{1}$ Chronic abdominal pain due to mesenteric ischemia caused by SMAD is rare. We report a case of type B ISMAD, which was treated conservatively with oral anticoagulants. Therapeutic options include conservative management as in the index case, endovascular management, or open surgical management using bypass or direct surgical reconstruction of the dissection. The treatment guidelines for ISMAD have not been established because of the rarity of the condition.

\section{Case report}

A 30-year-old male patient presented with abdominal pain and vomiting for three days. He was known to be diabetic and presented to the surgical outpatients' department with the complaint of abdominal pain, on and off for three months. He did not smoke or drink alcohol. There was no history of hematemesis or melena. On physical examination, the temperature was normal, pulse 110/ min, respiratory rate $18 / \mathrm{min}$, and blood pressure $140 / 86 \mathrm{mmHg}$. Minimal epigastric tenderness was elicited on palpation and bowel sounds were auscultated. Rectal examination was normal. Laboratory findings were normal with a hemoglobin of $14.3 \mathrm{~g} / \mathrm{dL}$, hematocrit $42 \%$, platelets $425,000 / \mathrm{mm}^{3}$, and white blood cell count $10,000 / \mathrm{mm}^{3}$. Serum analysis revealed the following: random blood sugar, $332 \mathrm{mg} /$ $\mathrm{dL}$; aspartate aminotransferase, 24U/L; alanine aminotransferase, 22U/L; and creatinine, $1.0 \mathrm{mg} / \mathrm{dL}$. Coagulation profile including PT with INR and aPTT were normal. An ultrasound of the abdomen revealed normal findings. We performed a contrast-enhanced computed tomography scan, which showed a small contrast-filled pouch in the proximal superior mesenteric artery (SMA) (Figure 1,2). However, all other branches showed normal vascularity and the SMA origin was normal. No thrombus was seen within the lumen of the vessel. 3D reconstruction imaging revealed mild enlargement of the SMA with a contrast-filled pouch (Figure 3). We treated the patient conservatively using low molecular weight heparin and tablet aspirin $150 \mathrm{mg}$ daily for anticoagulation. The patient improved and was discharged on warfarin. He was in followed up for six weeks without any problems, and then was lost to follow-up.

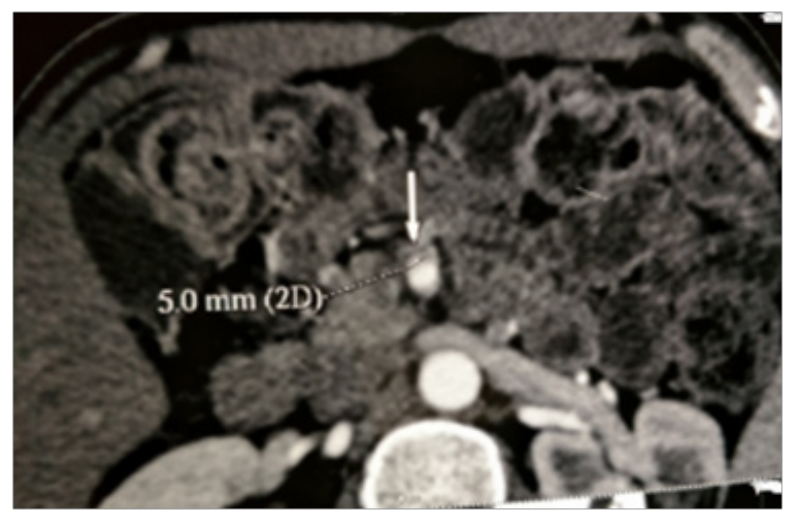

Figure I Axial, arterial-phase, contrast-enhanced, CT scan shows Small contrast filled pouch seen in the proximal Superior mesenteric artery.

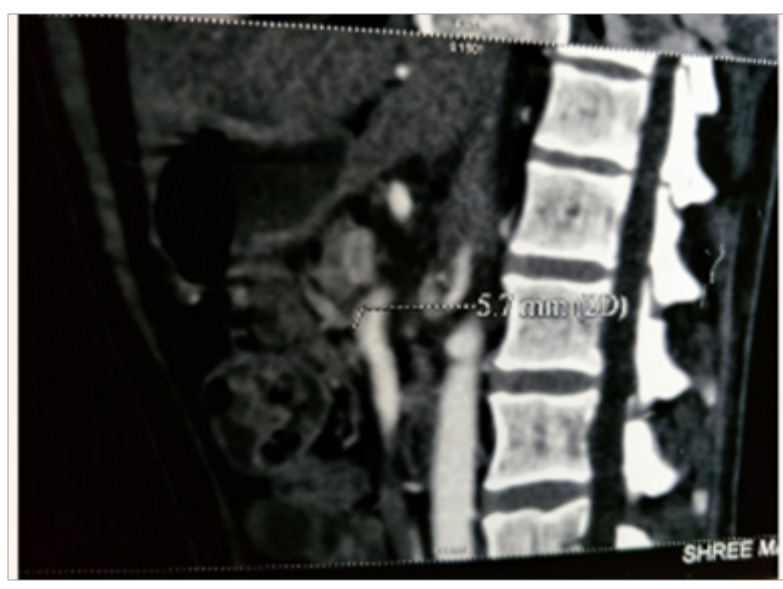

Figure 2 Sagittal, contrast-enhanced, arterial phase, maximum intensity projection CT scan shows a contrast filled pouch in the proximal superior mesenteric artery.

\section{Discussion}

ISMAD is rare. In a series of 6,666 autopsies, the incidence of SMAD was $0.06 \%$. Since Bauerfield et al first reported the disease 
in 1941, Luan et al described 296 patients with ISMAD up to 2013. Common clinical manifestations include acute abdominal pain, nausea, and vomiting. Ileus, melena, diarrhea and emaciation are other manifestations, while $18 \%$ of patients are asymptomatic. ${ }^{1}$ Chronic abdominal pain is rare in SMAD and is either due to dissection, vasospasm, inflammation, or mesenteric ischemia. However, 47 of the reported patients did not have any abdominal pain and the dissection was detected only incidentally. An extensive review of the literature by Subhas et al showed that ISMAD occurred more commonly in males. Most cases were reported in the fifth decade of life. ${ }^{2}$ Predisposing factors for SMAD include hypertension, smoking, cystic medial necrosis, vasculitis, fibromuscular dysplasia, atherosclerosis, ${ }^{3}$ and, in rare cases, strenuous exercise and trauma. The majority of cases of ISMAD were reported from Asia. This may indicate a genetic predisposition to SMAD in the Asian population. ${ }^{4}$ A classification based on the location of the SMAD, the Luan classification, ${ }^{5}$ is as follows:

I. Type A, the dissection was localized at the curved part of the SMA and extended proximally to the SMA ostium;

II. Type B, the dissection was limited to the curved part of the SMA;

III. Type $\mathrm{C}$, the dissection was localized at the curved part and extended distally, but the ileocolic artery or distal ileal artery was not involved; and

IV. Type D, the dissection was localized at the curved part and extended distally to the ileocolic artery or distal ileal artery.

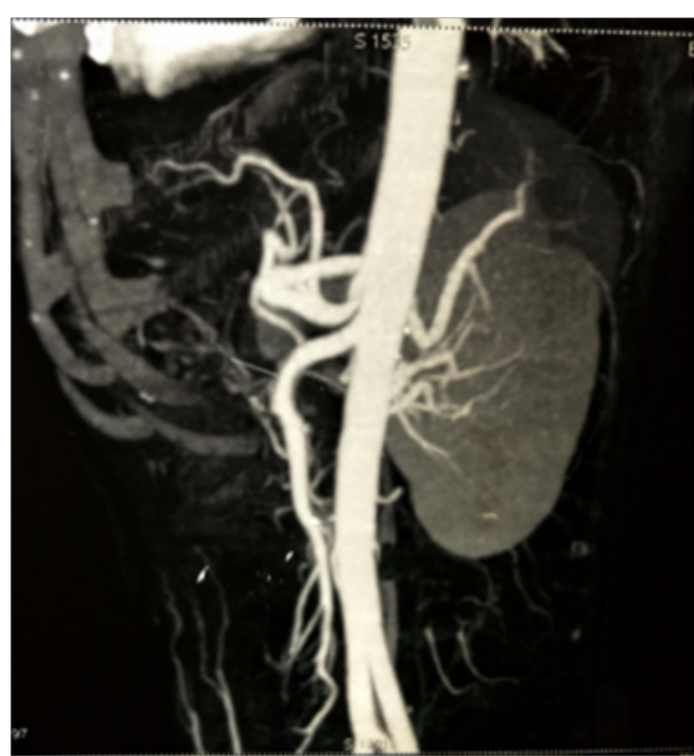

Figure 33 D reconstruction demonstrates an enlarged superior mesenteric artery lumen with contrast filled pouch.

SMAD may further be classified as grade I (peritonitis absent) and grade II (peritonitis present) ${ }^{6}$

Although dissection can be seen with the help of Doppler ultrasonography, computed tomography is the preferred imaging modality. Magnetic resonance angiography is an alternative examination, but it often fails to show the small details that computed tomography does. The asymptomatic patient can be managed conservatively. Endovascular intervention is recommended when aneurysmal dilation exceeds $2 \mathrm{~cm}$ in diameter, if there is bowel ischemia, or if there is compression of the lumen of greater than $80 \%$.

Treatment guidelines for ISMAD have not been established because of the rarity of the condition. For grade I ISMAD, most cases might be successfully treated with medical therapy, and endovascular stent placement appears to be an acceptable alternative if medical treatment fails. For grade II ISMAD, endovascular stenting combined with laparoscopic exploration and/or open surgery could be a reasonable option. ${ }^{8,9}$

\section{Conclusion}

ISMAD is rare. Computed tomography is the preferred imaging modality. Therapeutic options include conservative management as in the index case, endovascular management or open surgical management using bypass or direct surgical reconstruction of the dissection.

\section{Conflicts of interest}

There is no conflict of interest.

\section{Acknowledgments}

None.

\section{References}

1. Luan JY, Li X, Li TR, et al. Vasodilator and endovascular therapy for isolated superior mesenteric artery dissection and literature review. $J$ Vasc Surg. 2013;57(6):1612-1620.

2. Subhas G, Gupta A, Nawalany M, et al. Spontaneous isolated superior mesenteric artery dissection, a case report and literature review with management algorithm. Ann Vasc Surg. 2009;23(6):788-798.

3. Solis MM, Ranval TJ, McFarland DR, et al. Surgical treatment of superior mesenteric artery dissecting aneurysm and simultaneous celiac artery compression. Ann Vasc Surg. 1993;7(5):457-462.

4. Park UJ, Kim HT, Cho WH, et al. Clinical course and angiographic changes of spontaneous isolated superior mesenteric artery dissection after conservative treatment. Surg Today. 2014;44(11):2092-2097.

5. Luan JY, Li X. Computed tomography imaging features and classification of isolated dissection of the superior mesenteric artery. Eur J Vasc Endovasc Surg. 2013;46(2):232-235.

6. Dong $\mathrm{Z}, \mathrm{Fu} \mathrm{W}$, Chen B, et al. Treatment of symptomatic isolated dissection of superior mesenteric artery. J Vasc Surg. 2013;57(2 suppl):69S-76S

7. Min SI, Yoon KC, Min SK, et al. Current strategy for the treatment of symptomatic spontaneous isolated dissection of superior mesenteric artery. J Vasc Surg. 2011;54(2):461-466.

8. Dong Z, Fu W, Chen B, et al. Treatment of symptomatic isolated dissection of superior mesenteric artery. J Vasc Surg. 2013;57(2):69S-76S.

9. Wagenhäuser MU, Sagban TA, Witte M, et al. Isolated dissection of the superior mesenteric artery treated using open emergency surgery. World J Emerg Surg. 2014;9:47. 